\title{
Aubry-Mather Sets and Birkhoff's Theorem for Geodesic Flows on the Two-Dimensional Torus
}

\section{L. Bialy}

Department of Theoretical Mathematics, The Weizmann Institute of Science, Rehovot 76100, Israel

\begin{abstract}
In this paper we state the graph property for incompressible continuouse tori invariant under goedesic flows of Riemannian metrics on the two-dimensional torus. Also our method gives a new proof of Birkhoff's theorem for twist maps of the cylinder. We prove that if there exist an invariant incompressible torus of geodesic flow with irrational rotation number then it necessarily contains the Aubry-Mather set with this rotation number.
\end{abstract}

\section{Introduction}

One theorem of Birkhoff states that any continuous simple closed incontractible curve which is invariant under a twist symplectomorphism of the cylinder $\mathscr{C}=S^{1} \times \mathbb{R}$ is the graph of a continuous function on $S^{1}$ (see [H1, H2]). The purpose of this paper is to use a variational approach for this problem which allows one to prove an analogous theorem for geodesics on the two-dimensional torus.

Let $T^{*} \mathbb{T}^{2}$ be the contangent bundle of the 2-torus $\mathbb{T}^{2}=\mathbb{R}^{2} / \mathbb{Z}^{2}, \theta: T^{*} \mathbb{T}^{2} \rightarrow \mathbb{T}^{2}$ the natural projection. Let $\omega^{2}=d \omega^{1}$ be the natural symplectic structure on $T^{*} \mathbb{T}^{2}$, where $\omega^{1}$ is the canonical 1 -form on $T^{*} \mathbb{T}^{2}$. Let $H: T^{*} \mathbb{T}^{2} \rightarrow \mathbb{R}$ be a smooth hamiltonian function on $T^{*} \mathbb{T}^{2}, h_{t}$ the corresponding hamiltonian flow. Throughout this paper we assume that $H$ is strictly convex on the fibres of $T^{*} T^{2}$ and is symmetric,

$$
H\left(x, p_{x}\right)=H\left(x,-p_{x}\right) \text { for all } x \in \mathbb{T}^{2}, \quad p_{x} \in T_{x}^{*} \mathbb{T}^{2} .
$$

Let $M=\left\{\left(x, p_{x}\right) \in T^{*} \mathbb{T}^{2}: H\left(x, p_{x}\right)=h\right\}$ for a fixed $h>\max _{x \in \mathbb{T}^{2}} H(x, 0)$. It is easily seen that $M$ is diffeomorphic to $\mathbb{T}^{3}$. Let $l: L \subset M$ be a topological 2-torus in $M, l$ is a continuous embedding. We will call $L$ incompressible if $l_{*}: \pi_{1}(L) \rightarrow \pi_{1}(M)$ is a monomorphism.

Theorem 1.1. Let $\imath: L \subset M$ be a continuously embedded incompressible invariant 2-torus of the hamiltonian flow $h_{t}$. If $L$ has no closed orbits of the flow then $L$ is a continuous cross-section of the cotangent bundle $T^{*} \mathbb{T}^{2}$. 
In the case when $L$ is smooth $\left(C^{3}\right)$ this theorem was proved in [B-P2] (see also [B]). Let us note that in that case the flow $h_{t}$ on $L$ is necessarily transitive. In this paper we are free from this restriction.

Let us outline our approach to this theorem. Let $\hat{H}$ be the function which is homogeneous of degree two on the fibres of $T^{*} T^{2}$ and coincides with $H / h$ on $M$, $\hat{h}_{t}$ the corresponding hamiltonian flow. It is clear that the trajectories of the flows $h_{t}$ and $\hat{h}_{t}$ in $M$ coincide up to parametrization, and $L$ is invariant under the flow $\hat{h}_{t}$. Therefore we will assume throughout the rest of this paper that $H$ is homogeneous of degree 2 on the fibres of $T^{*} T^{2}$. It follows from the conditions mentioned above that the Legendre transformation of $H$ determines a symmetric Finsler metric $F$ on $\mathbb{T}^{2}$. In addition, projections on $\mathbb{T}^{2}$ of the trajectories of the flow $h_{t}$ in $M$ are geodesics of this Finsler metric. The metric $F$ defines a $\mathbb{Z}^{2}$-periodic Finsler metric on the covering plane $\mathbb{R}^{2}$.

Definition 1.2. (see [Hed]). A geodesic on $\mathbb{T}^{2}=\mathbb{R}^{2} / \mathbb{Z}^{2}$ is called an $A$-geodesic if each of its liftings to the covering plane minimizes the $F$-length between any two of its points.

Our method is based on Aubry-Mather theory for twist symplectomorphisms of the cylinder (see [A], [M]) which was applied in [B-P1], [Ba] for geodesics on the two-dimensional torus. More precisely, we show that the projections on $\mathbb{T}^{2}$ of the trajectories lying on $L$ are $A$-geodesics. To show this we use a classical property of Poincaré rotation number and arguments of contact geometry. Then the results of [B-P1] yield the proof of our main theorem.

The paper is organized as follows. In Sect. 2 we construct a cross-section for the flow on $L$. In Sect. 3 we prove a theorem about intersections of geodesics with the same rotation number. In Sect. 4 we study the linking number of legendrian curves. We complete the proof of the main theorem in Sect. 5.

\section{Cross-Section for the Flow on $L$}

First of all we will obtain the following "niceness" condition of the topological embedding of $L$ into $M$.

Lemma 2.1. If Lis a continuously embedded invariant 2-torus of the flow in $M$, then $L$ is locally flat in $M$. That means that for each point of $L$ there is a neighbourhood $U$ in $M$ such that $(U, U \cap L)$ is homeomorphic to $\left(\mathbb{R}^{3}, \mathbb{R}^{2}\right)$.

Proof. Let $s \operatorname{grad} H$ denote the hamiltonian vector field in $M$. For each point of $L$ there is a neighbourhood $U$ in $M$ such that $U \cap L$ is homeomorphic to $\mathbb{R}^{2}$ and there exists a diffeomorphisms $f$ of $C l(U)$ to the standard 3-cube $I^{3}=$ $\left\{\left(x_{1}, x_{2}, x_{3}\right) \in \mathbb{R}^{3}:\left|x_{i}\right| \leqq 1\right\}$ taking $s \operatorname{grad} H$ to the field $\partial / \partial x_{1}$. Let $I^{2}=\left\{x \in I^{3}: x_{1}=-1\right\}$. Then $f(C l(U) \cap L) \cap I^{2}$ is a simple continuous arc in $I^{2}$. It follows from Jordan-Schönflies arguments that this arc is locally flat in $I^{2}$, and hence $f(U \cap L)$ is locally flat in $I^{3}$. This completes the proof of the lemma.

Remark 2.2. It follows from Lemma 2.1 and a theorem of Brown (see $[\mathrm{R}]$ ) that $L$ is bicollared in $M$, i.e. there exists a neighbourhood of $L$ in $M$ homeomorphic to 
$L \times(-\varepsilon, \varepsilon)$. Then it is easily seen that in this neighbourhood $L$ can be approximated by a smoothly embedded torus, homotopic to $L$ (this follows for instance from Theorem 6.3 of $[\mathrm{Mu}]$ ).

Let $q_{1} \bmod 1, q_{2} \bmod 1$ be the standard coordinates on $\mathbb{T}^{2}=\mathbb{R}^{2} / \mathbb{Z}^{2}, p_{1}, p_{2}$ conjugate momenta. Let $B$ be an incompressible 2-torus in $M$ defined by

$$
B=\left\{x \in T^{*} \pi^{2}: H_{p_{2}}(x)=0, H_{p_{1}}(x)>0\right\} \cap M .
$$

We will use the following proposition from [B-P2].

Proposition 2.3. If $L$ is incompressible invariant torus in $M$ then $L$ is homologous to $B$ in $M$.

Let us also define an incompressible 2-torus $S$ in $M$ by $S=\{x \in M: \theta(x) \in e\}$, where $e$ is a closed $A$-geodesic on $\mathbb{T}^{2}$ which is the shortest in the free homotopy class $(0,1) \in \mathbb{Z}^{2}$. Let $e_{+}, e_{-}$be the closed orbits of the flow $h_{t}$ in $M$ corresponding to $e, e_{+}, e_{-} \subset S$. Let $\sigma: T^{*} \mathbb{T}^{2} \rightarrow T^{*} \mathbb{T}^{2}$ be the involution taking a point $\left(x, p_{x}\right)$ to $\left(x,-p_{x}\right)$. Since $H$ is symmetric, then $\sigma(L)$ is an invariant torus of the hamiltonian flow.

\section{Theorem 2.4.}

i. The intersection set $\gamma=L \cap S$ is a simple closed curve.

ii. This curve $\gamma$ is two-sided on $L$ and trajectories of the flow $h_{t}$ on $L$ pass from one side of $\gamma$ to the other. In addition, $\gamma$ is not null-homotopic on L nor on $S$.

iii. The invariant tori $L$ and $\sigma(L)$ do not intersect.

Proof. It follows from Proposition 2.3 that $L \cap S \neq \varnothing$, since $L$ and $S$ are not homologous in $M$ and both of them are incompressible. The hamiltonian vector field $s \operatorname{grad} H$ in $M$ is everywhere transversal to $S$ outside the closed orbits $e_{+}, e_{-}$ and $(L \cap S) \cap\left(e_{+} \cup e_{-}\right)=\varnothing$. This implies that $L \cap S$ is a one-dimensional topological submanifold consisting of a finite number of simple non-intersecting closed curves. Each of them is two-sided in $L$ and trajectories of the flow on $L$ pass from one of its sides to the other, since $S$ is two-sided in $M$ and trajectories of the flow on $L$ are transversal to $S$. It follows from Poincaré-Bendixson arguments that each of these curves is not null-homotopic on $L$, since the flow $h_{t}$ has no fixed points on $L$ (see $[\mathrm{Be}],[\mathrm{C}-\mathrm{L}]$ ). Therefore each of these curves is not null-homotopic also on $S$, since $L$ is incompressible. Let us show that $L \cap S$ consists of only one simple closed curve $\gamma$. Let us fix an orientation $\omega^{3}$ on $M$ defined by $d H \wedge \omega^{3}=\omega^{2} \wedge \omega^{2}$ and some orientation $s$ on $S$. The closed orbits $e_{+}, e_{-}$divide $S$ into two connected domains $S_{+}$and $S_{-}$. In addition, the transversal orientation $\omega^{2}$ by the hamiltonian vector field coincides with $s$ on $S_{+}$and with $-s$ on $S_{-}$. Also $S_{-}=\sigma\left(S_{+}\right)$.

Lemma 2.5. Let $\gamma^{1}, \gamma^{2}$ be two curves from $L \cap S$ lying in $S_{+}$(or $\left.S_{-}\right)$. Then $\gamma^{1}, \gamma^{2}$ coincide.

Proof. Let $\tilde{\gamma}^{1} \subset S_{+}\left(S_{-}\right)$be a smooth simple closed curve sufficiently closed to $\gamma^{1}$. Let $\tau: \gamma^{1} \rightarrow \mathbb{R}$ be a continuous function such that $h_{\tau(x)} x \in \gamma^{2}$ for all $x \in \gamma^{1}$. It is easily seen that there exists a smooth function $\tilde{\tau}: \tilde{\gamma}^{1} \rightarrow \mathbb{R}$ such that $h_{\tilde{\tau}(x)} x \in \tilde{\gamma}^{2}$ for all $x \in \tilde{\gamma}^{1}$, where $\tilde{\gamma}^{2}$ is a smooth simple closed curve close to $\gamma^{2}$. In addition, $\tilde{\gamma}^{1}, \tilde{\gamma}^{2}$ lie 
in $S_{+}\left(S_{-}\right)$and do not intersect. We have then

$$
\int_{\tilde{\gamma}^{1}} \omega^{1}=\int_{\tilde{\gamma}^{2}} \omega^{1} \text { and hence } \int_{\Gamma} \omega^{2}=0
$$

where $\Gamma \subset S_{+}\left(S_{-}\right)$is the domain bounded by the curves $\tilde{\gamma}^{1}, \tilde{\gamma}^{2}$. This contradicts the fact that $\omega^{2}$ does not vanish on $S_{+}\left(S_{-}\right)$. This completes the proof of the lemma.

It follows from Lemma 2.5 that $L \cap S$ consists of at most two different curves $\gamma_{+}$and $\gamma_{-}, \gamma_{+} \subset S_{+}, \gamma_{-} \subset S_{-}$. Let $L^{1}, L^{2}$ be the two domains of $L$ bounded by the curves $\gamma_{+}, \gamma_{-}$. It is easily seen that for each point $P$ from $L^{1}$ there is a $T>0$ such that $h_{T} P \in L^{1}$ and the segment $h_{t} P, t \in[0, T]$ crosses $\gamma_{+}$and $\gamma_{-}$only once. Let $\delta$ be a simple closed curve consisting of the segment $h_{t} P, t \in[0, T]$ and a simple arc lying in $L^{1}$ joining the ends of this segment. Then $\delta$ is not null-homotopic and is not homotopic to $\gamma_{ \pm}$on $L$. On the other hand the intersection number $\delta \circ S$ equals zero, since $\gamma_{+} \subset S_{+}$and $\gamma_{-} \subset S_{-}$. Hence $\delta$ is necessarily homotopic to $\gamma_{ \pm}$. This contradiction yields the proof of $i$. Now it is very simple to show iii. Indeed if $L \cap \sigma(L) \neq \varnothing$, then $\gamma \cap \sigma(\gamma) \neq \varnothing$. But $\gamma$ and $\sigma(\gamma)$ lie in the different domains $S_{+}, S_{-}$. This completes the proof of the theorem.

\section{Intersection of Recurrent Geodesics with the Same Rotation Number}

Let $h: \gamma \rightarrow \gamma$ be the return map of the flow $h_{t}$ on $L$. It follows from Theorem 2.4 that $h$ is a well defined homeomorphism of the circle $\gamma$. Let $\omega$ be the Poincare rotation number of $h$, which is irrational, since $h_{t}$ has no closed orbits on $L$.

Definition 3.1. A point $P$ in $M$ is said to be recurrent if $P$ belongs to the $\omega$-limit set of the trajectory $h_{t} P$.

Let $\gamma_{\omega}$ be the set of all recurrent points of $\gamma, L_{\omega}$ the set of all recurrent points of $L$. It is clear that $\gamma_{\omega}$ is the minimal set of the homeomorphism $h$ and $L_{\omega}$ consists of the trajectories passing through $\gamma_{\omega}$.

Let $T^{*} \mathbb{R}^{2}$ be the cotangent bundle of the covering plane $\mathbb{R}^{2}$ with coordinates $q_{1}, q_{2}$, and $\eta: T^{*} \mathbb{R}^{2} \rightarrow \mathbb{R}^{2}$ be the natural projection. Let $\xi: T^{*} \mathbb{R}^{2} \rightarrow T^{*} \mathbb{T}^{2}, \zeta: \mathbb{R}^{2} \rightarrow \mathbb{T}^{2}$ be the natural covering maps, $T_{1}, T_{2}$ be the unit shifts of $T^{*} \mathbb{R}^{2}$ along the axes $q_{1}$, $q_{2}$ respectively, $T_{i} \xi=\xi T_{i}(i=1,2)$. Let us lift $L$ to the covering space $T^{*} \mathbb{R}^{2}$. It follows easily from Proposition 2.3 that $\xi^{-1}(L)$ is homeomorphic to $\mathbb{R}^{2}$, also $\xi^{-1}(L)$ is invariant under the flow with the hamiltonian function $H \circ \xi$. Let $\gamma_{0}$ be some lifting of $\gamma$ to $\xi^{-1}(L), \gamma_{n}=\left(T_{1}\right)^{n} \gamma_{0} \quad(n \in \mathbb{Z})$. It is easily seen that $\gamma_{i}(i \in \mathbb{Z})$ is homeomorphic to $\mathbb{R}$ and $\gamma_{i} \cap \gamma_{j}=\varnothing$ for $i \neq j$, also $T_{2} \gamma_{i}=\gamma_{i}$. We may assume without loss of generality that the trajectories of the flow on $\xi^{-1}(L)$ cross $\gamma_{n}$ in such a way that $n$ increases. Otherwise we will change the direction of the $q_{1}$-axis. Let $e_{n}$ be the different liftings to $\mathbb{R}^{2}$ of the $A$-geodesic $e$ on $\mathbb{T}^{2}$ (see Sect. 2), $e_{n}=\eta\left(\gamma_{n}\right)$. Let $\Omega$ be the $F$-length of $e$ on $\mathbb{T}^{2}$. Let us recall now some facts about $A$-geodesics on $\mathbb{T}^{2}$ from [Hed] (see also [B-P1], [Ba]).

3.2. Each $A$-geodesic on the covering plane has no self-intersection points. Two different $A$-geodesics on the covering plane intersect in at most one point and do not intersect if they are asymptotic. 
3.3. For each $A$-geodesic $g$ on the covering plane there are two parallel lines defined by $\alpha q_{1}+\beta q_{2}=\delta_{i}(i=1,2)$ such that $g$ lies in the strip between them and crosses each arc connecting them. The ratio $s=-\beta / \alpha$ is called the type of this $A$-geodesic.

Let $K_{s}$ be the set of all $A$-geodesics of the type $s$, where $s$ is assumed to be irrational. We assume also that each $A$-geodesic $g(t)$ from $K_{s}$ is parametrized by $F$-length such that $q_{1}(g(t)) \rightarrow+\infty$ when $t \rightarrow+\infty$. Let $\Gamma_{s}$ be the set of all $A$-geodesics from $K_{s}$ such that the corresponding trajectories of the flow $h_{t}$ in $M$ are recurrent. Let $\varphi: \mathbb{R} \rightarrow e_{0}$ be the $F$-length parametrization of the $A$-geodesic $e_{0}$ such that $q_{2}(\varphi(t)) \rightarrow+\infty$ when $t \rightarrow+\infty, e_{n}(t)=\left(T_{1}\right)^{n} \varphi(t)$ the $F$-length parametrization of $e_{n}$. Each $A$-geodesic $g$ on $\mathbb{R}^{2}$ of the type $s$ intersects $e_{n}$ at unique point, $g \cap e_{n}=e_{n}\left(u_{n}(g)\right)$.

Definition 3.4. (see [A]). A pair of functions $f^{+}, f^{-}: \mathbb{R} \rightarrow \mathbb{R}$ is called a pair of $h$-functions if

a. $f^{+}, f^{-}$are strictly increasing and $f^{ \pm}(x+\Omega)=f^{ \pm}(x)+\Omega$ for all $x \in \mathbb{R}$.

b. $f^{+}$is continuous from the right and $f^{-}$is continuous from the left and

$$
f^{+}(x-0)=f^{-}(x), \quad f^{-}(x+0)=f^{+}(x) .
$$

The following theorem from [B-P1] (see also [Ba]) will be crucial in the main result of this section.

Theorem 3.5. Suppose $s$ is irrational. Then

i. $K_{s}$ is ordered, that means that two different A-geodesics from $K_{s}$ do not intersect.

ii. There exists a pair of h-functions $f^{+}, f^{-}$such that for each A-geodesic $g$ from $\Gamma_{s}, u_{n}(g)=f^{*}(n s \Omega+\alpha)$ for some $\alpha \in \mathbb{R}$ and $* \in\{+,-\}$. Conversely, for each $\alpha \in \mathbb{R}$ and $* \in\{+,-\}$ one can find $g$ from $\Gamma_{s}$ such that $u_{n}(g)=f^{*}(n s \Omega+\alpha)$.

iii. If $f^{+}(\alpha) \neq f^{-}(\alpha)$ then the A-geodesics $g^{+}, g^{-}$corresponding to the configurations $f^{+}(n s \Omega+\alpha), f^{-}(n s \Omega+\alpha)$ are asymptotic.

iv. If $f^{+}=f^{-}\left(f^{ \pm}\right.$are continuous $)$then $K_{s}=\Gamma_{s}$ and through each point of $\mathbb{R}^{2}$ passes some A-geodesic from $K_{s}$.

Now we are able to state the main result of this section. Let $G_{\omega}$ be the subset of $M$ consisting of all the points $P$ such that $P$ is recurrent and the geodesics $\zeta^{-1} \theta\left(h_{t} P\right)$ in $\mathbb{R}^{2}$ belong to $\Gamma_{\omega}(\omega$ is defined in the beginning of the section).

Theorem 3.6. Either $G_{\omega}$ coincides with $L_{\omega}$ and then each trajectory of $L_{\omega}$ projects to an A-geodesic on $\mathbb{T}^{2}$. Or $G_{\omega} \cap L=\varnothing$ and there exists a pair of geodesics on $\mathbb{T}^{2}$ $\theta\left(h_{t} P\right)$ and $\theta\left(h_{t} O\right)$ for $P \in L_{\omega}, O \in G_{\omega}$ such that some of their liftings to the covering plane $\mathbb{R}^{2}$ cross infinitely often.

Proof. The sets $L, L_{\omega}, G_{\omega}$ are invariant under the flow $h_{t}$. If $L \cap G_{\omega} \neq \varnothing$ then $L_{\omega}$ and $G_{\omega}$ coincide, since each trajectory lying in $L_{\omega} \cap G_{\omega}$ is everywhere dense in $L_{\omega}$ and $G_{\omega}$. Now let us assume that $L_{\omega} \cap G_{\omega}=\varnothing$. Then for each point $P \in L_{\omega}$ each lifting to $\mathbb{R}^{2}$ of the geodesic $\theta\left(h_{t} P\right)$ does not belong to $\Gamma_{\omega}$. Let $l(t)$ be some lifting to $\mathbb{R}^{2}$ of $\theta\left(h_{t} P\right)$. Let us note that $l(t)$ necessarily intersects some $A$-geodesic $g$ from $\Gamma_{\omega}$. Indeed, otherwise $l(t)$ lies in the gap between two asymptotic $A$-geodesics $g^{+}$, $g^{-}$from $\Gamma_{\omega}$ (see Theorem 3.5). But then it follows from Theorems 2.4, 3.5 that $l(t)$ is asymptotic to $g^{+}, g^{-}$and hence the trajectory $h_{t} P$ is not recurrent. Thus $l$ 
transversally intersects with some $A$-geodesic $g$ from $\Gamma_{\omega}$. Let $O \in G_{\omega}$ be such that $g$ is the lifting to $\mathbb{R}^{2}$ of the geodesic $\theta\left(h_{t} O\right)$. We will assume without loss of generality that $\theta(O)=\theta(P)$. Since $P \in L_{\omega}$ then for each $\varepsilon>0$ there is a sequence $t_{k} \rightarrow+\infty$ such that $h_{t_{k}} P$ belongs to the $\varepsilon$-neighbourhood $U$ of $P$ in $M$. This implies that if $\varepsilon$ is sufficiently small then the geodesic $\zeta(l)=\theta\left(h_{t} P\right)$ will cross again $\zeta(g)=\theta\left(h_{t} O\right)$ on $\mathbb{T}^{2}$ at some points close to $\theta\left(h_{t_{n}} P\right)$. On the covering plane that means that $l(t)$ crosses $A$-geodesics $g_{m_{k} n_{k}}=\left(T_{1}\right)^{m_{k}}\left(T_{2}\right)^{n_{k}} g$. Here $\left(m_{k}, n_{k}\right) \in \mathbb{Z}^{2}$ is the homotopy class of the closed curve on $T^{2}$ consisting of the segment $\theta\left(h_{t} P\right), t \in\left[0, t_{k}\right]$, and a small arc lying in $\theta(U)$ joining the ends of this segment.

Lemma 3.7. There are two infinite subsets $K_{1}, K_{2}$ of $\mathbb{Z}_{+}$such that

$$
n_{k} / m_{k}<\omega \text { for } k \in K_{1}, \quad n_{k} / m_{k}>\omega \text { for } k \in K_{2} \text {. }
$$

Proof. This fact is the main property of the Poincare rotation number and follows easily from the fact that the homeomorphism $h: \gamma \rightarrow \gamma$ is topologically conjugate to the rotation on the minimal set $\gamma_{\omega}$. This completes the proof the lemma.

It follows from Theorem 3.5 that the geodesics $g_{m_{i} n_{i}}$ and $g_{m_{j} n_{j}}$ lie on different sides of $g$ for all $i \in K_{1}, j \in K_{2}$. And hence $l$ intersects $g$ infinitely often. This completes the proof of the theorem.

\section{Linking of Trajectories}

In this section we apply methods of contact geometry to prove the following

Theorem 4.1. The sets $L_{\omega}$ and $G_{\omega}$ coincide.

The proof will be given later. Let $Q$ be the manifold of all oriented contact elements of $\mathbb{T}^{2}$ with the natural contact structure $q$ (see [Ar, p. 354]). Let $\Phi: M \rightarrow Q$ be the map taking $w \in M$ to the oriented contact element at the point $\theta(w)$ generated by the vector $\theta_{*} s \operatorname{grad} H(w)$. It is easily seen that $\Phi$ is a diffeomorphism. Let $\Phi_{*}^{-1}(q)$ be the induced contact structure in $M$. Planes of this contact structure are generated by the hamiltonian field in $M$ and a vertical field $v$ tangent to the fibres of the bundle $\theta: M \rightarrow \mathbb{T}^{2}$ (see [B-P2]). Let us identify $M$ with $Q$ via the diffeomorphism $\Phi$. Let $N=\mathbb{T}^{2} \times \mathbb{R}, \tau: N \rightarrow M$ be the natural covering map taking a point $(x, \varphi) \in N$, $x \in \mathbb{T}^{2}, \varphi \in \mathbb{R}$ to the oriented contact element in $T_{x} \mathbb{T}^{2}$ having the oriented angle $\varphi \bmod 2 \pi$ with the vector $\partial / \partial q_{1}$. Let us note that $q_{1} \bmod 1, q_{2} \bmod 1, \varphi \bmod 2 \pi$ are coordinates in $M$ and the torus $B$ in $M$ is defined by $\varphi=0$. Since $L$ is homologous to $B$ in $M$ we have that each lifting of $L$ to $N$ is a 2-torus homologous to $\mathbb{T}^{2} \times\{0\}$ in $N$. Let $\tilde{\theta}: N \rightarrow \mathbb{T}^{2}$ be the natural projection. In a natural way we identify the configuration space $\mathbb{T}^{2}$ with $B$ in $M$ and $\mathbb{T}^{2} \times\{0\}$ in $N$. Let us lift the contact structure $q$ from $M$ to $N$.

Defintion 4.2. A smooth curve is called legendrian if it is tangent to the contact structure.

Definition 4.3. Two simple closed non-intersecting curves in $N$ are said to be unlinked if there is a locally flat topologically embedded torus in $N$ homologous to $\mathbb{T}^{2} \times\{0\}$ separating these two curves. 
Let $\tilde{\sigma}: N \rightarrow N$ be the map taking $(x, \varphi)$ to $(x, \varphi+\pi)$ for all $x \in \mathbb{T}^{2}, \varphi \in \mathbb{R}$. The map $\tilde{\sigma}$ covers the involution $\sigma$ of $M$.

Theorem 4.4. Let $C_{1}, C_{2}$ be two smooth simple closed legendrian curves in $N$, such that the restrictions $\left.\widetilde{\theta}\right|_{C_{1}},\left.\widetilde{\theta}\right|_{C_{2}}$ are diffeomorphisms. Let us assume also that the curves $\tilde{\theta}\left(C_{1}\right), \tilde{\theta}\left(C_{2}\right)$ are transversal on $\mathbb{T}^{2}$. If the curves $\tilde{\sigma}^{m} C_{1}$ and $\tilde{\sigma}^{n} C_{2}$ are unlinked for all integers $m$ and $n$, then

$$
\operatorname{card}\left(\tilde{\theta}\left(C_{1}\right) \cap \tilde{\theta}\left(C_{2}\right)\right)=\left|\tilde{\theta}\left(C_{1}\right) \circ \tilde{\theta}\left(C_{2}\right)\right| .
$$

The proof will be given later. Let us introduce the following notations. Let $N^{E}=\mathbb{T}^{2} \times[-E, E], N^{E+}=\mathbb{T}^{2} \times[+E,+\infty), N^{E-}=\mathbb{T}^{2} \times(-\infty,-E]$. Let $C_{1}, C_{2}$ be two oriented smooth simple closed curves in $N$. Let us choose $E$ sufficiently large to have $C_{1}, C_{2} \subset N^{E}$. Let $\Sigma^{i+}, \Sigma^{i-}(i=1,2)$ be 2 -chains such that

$$
\partial \Sigma^{i+}=C_{i}\left(\bmod N^{E+}\right) \text { and } \partial \Sigma^{i-}=C_{i}\left(\bmod N^{E-}\right) \text { for } i=1,2 .
$$

Then we define the four numbers

$$
\begin{array}{ll}
l^{+}\left(C_{1}, C_{2}\right)=\Sigma^{1+}{ }_{\circ} C_{2}, & l^{+}\left(C_{2}, C_{1}\right)=\Sigma^{2+}{ }_{\circ} C_{1}, \\
l^{-}\left(C_{1}, C_{2}\right)=\Sigma^{1-}{ }_{\circ} C_{2}, & l^{-}\left(C_{2}, C_{1}\right)=\Sigma^{2-}{ }^{\circ} C_{1} .
\end{array}
$$

It is easily seen that these numbers are well defined and coincide with the linking number in $\mathbb{T}^{2} \times \mathbb{R}$ when $C_{1}, C_{2}$ are homologous (see [B-P2, Sect. 3]).

Lemma 4.5. Let $\lambda$ be a locally flat topological torus in $\operatorname{int}\left(N^{E}\right)$ homologous to $\mathbb{T}^{2} \times\{0\}$. Then

i. $\lambda$ divides $N^{E}$ into two connected components $N_{+}, N_{-}$such that

$$
N_{+} \cap N_{-}=\lambda, \quad \partial N_{ \pm}=\lambda \cup\left(\mathbb{T}^{2} \times\{ \pm E\}\right) .
$$

ii. Each of $N_{+}, N_{-}$is homeomorphic to $N^{E}$.

iii. If $C_{1}, C_{2}$ are two smooth unlinked simple closed curves such that

$$
C_{1} \subset N_{+}, \quad C_{2} \subset N_{-} \text {then } l^{+}\left(C_{1}, C_{2}\right)=l^{-}\left(C_{2}, C_{1}\right)=0 \text {. }
$$

Proof.

i. Easily follows from Jordan-Brouwer arguments and the fact that $\lambda$ is homologous to $\mathbb{T}^{2} \times\{0\}$.

ii. Since $\lambda$ is locally flat then $\lambda$ is bicollared in $N^{E}$ (see [R]). Then it follows from [E] that $N_{+}, N_{-}$are homeomorphic to $N^{E}$.

iii. If $C_{1}$ lies in $N_{+}$then it is easily seen that there exists a 2-chain $\Sigma^{1+}$ not intersecting $C_{2}$ (see Remark 2.2). Hence $l^{+}\left(C_{1}, C_{2}\right)=0$ and analogously $l^{-}\left(C_{2}, C_{1}\right)=0$. This completes the proof of the the lemma.

For a smooth oriented closed curve $a$ on $\mathbb{T}^{2}$ let us denote by $\operatorname{leg}(a)$ the closed curve in $M$ formed by the oriented contact elements $\dot{a}$ which are tangent to $a$ and positively directed (recall that $M$ and $Q$ are identified).

Proof of Theorem 4.4. Let $a_{i}=\tilde{\theta}\left(C_{i}\right), C_{i, m}=\tilde{\sigma}^{m}\left(C_{i}\right)$ for $i=1,2$ and $m \in \mathbb{Z}$. Since $\left.\tilde{\theta}\right|_{C_{i}}$ is a diffeomorphism then $C_{i}$ is the graph of a smooth function $\varphi_{i}: a_{i} \rightarrow \mathbb{R}$ :

$$
C_{i}=\left\{(x, \varphi) \in N: x \in a_{i} \text { and } \varphi=\varphi_{i}(x)\right\} .
$$


Also the curve $C_{i, m}$ is the graph of the function $\varphi_{i, m}=\varphi_{i}+m \pi$. Since $C_{i}$ is a legendrian curve, one can assume also that $C_{i}$ are oriented in such a way that $\operatorname{leg}\left(a_{i}\right)=\tau\left(C_{i}\right)$. That means that $\varphi_{i}(x) \bmod 2 \pi$ equals the oriented angle between the vectors $\partial / \partial q_{1}$ and $\dot{a}_{i}$ in $T_{x} \mathbb{T}^{2}$. We can assume also without loss of generality that $a_{1} \circ a_{2} \geqq 0$.

Lemma 4.6. The numbers $l^{+}, l^{-}$satisfy the following relations

$$
\begin{gathered}
l^{+}\left(C_{1, m}, C_{2, n}\right)-l^{-}\left(C_{1, m}, C_{2, n}\right)=a_{1} \circ a_{2}, \\
l^{+}\left(C_{1, m}, C_{2, n}\right)=l^{-}\left(C_{2, n}, C_{1, m}\right), \quad l^{+}\left(C_{2, n}, C_{1, m}\right)=l^{-}\left(C_{1, m}, C_{2, n}\right) .
\end{gathered}
$$

Proof. The proof easily follows from the definitions given above. Let us prove the first one for example. Let $\Sigma^{1, m+}$ and $\Sigma^{1, m-}$ be 2-chains defined by

$$
\begin{aligned}
& \Sigma^{1, m+}=\left\{(x, \varphi) \in N^{E}: x \in a_{1}, \varphi_{1, m}(x) \leqq \varphi \leqq E\right\}, \\
& \Sigma^{1, m-}=\left\{(x, \varphi) \in N^{E}: x \in a_{1},-E \leqq \varphi \leqq \varphi_{1, m}(x)\right\}
\end{aligned}
$$

and oriented in such a way that $\partial \Sigma^{1, m+}=C_{1, m}\left(\bmod N^{E+}\right), \partial \Sigma^{1, m-}=$ $C_{1, m}\left(\bmod N^{E-}\right)$. Then $\left(\Sigma^{1, m+}-\Sigma^{1, m-}\right) \circ C_{2, n}=a_{1} \circ a_{2}$. This implies the relation.

Let $D=a_{1} \cap a_{2}$. For each point $A \in D$ let $\Delta(A)=\varphi_{2}(A)-\varphi_{1}(A)$ and $\mu(A), v(A)$ be such that $\Delta(A)=\mu(A) \pi+v(A)$, where $\mu(A) \in \mathbb{Z}, v(A) \in(0, \pi)$. Let $\mu_{+}=\max \mu(A)$, $\mu_{-}=\min _{A \in D} \mu(A)$. Let $D_{\mu}=\{A \in D: \mu(A)=\mu\}$ and $d_{\mu}=\operatorname{card} D_{\mu}, d=\operatorname{card} D$, where $\mu \in\left[\mu_{-}, \mu_{+}\right] \cap \mathbb{Z}$. To prove the theorem we have to show that card $D=a_{1} \circ a_{2}$. Let us consider two cases

1. $a_{1} \circ a_{2}=0$. In this case it follows from Lemma 4.6 that

$$
l^{+}\left(C_{1, m}, C_{2, n}\right)=l^{-}\left(C_{1, m}, C_{2, n}\right)=l^{+}\left(C_{2, n}, C_{1, m}\right)=l^{-}\left(C_{1, m}, C_{2, n}\right)
$$

Since $C_{1, m}$ and $C_{2, n}$ are unlinked then it follows from Lemma 4.5 that these numbers equal zero. On the other hand it is easily seen that

$$
l^{+}\left(C_{1, \mu+}, C_{2}\right)=\sum_{A \in D_{\mu+}}(-1)^{\mu+}=(-1)^{\mu+} d_{\mu+},
$$

hence $D_{\mu+}=\varnothing$ and then $D=\varnothing$.

2. $a_{1} \circ a_{2}>0$. In this case the following lemma allows us to reduce the problem to the case $a_{1} \circ a_{2}=1$.

\section{Lemma 4.7.}

i. There exists a covering map $\mathbb{T}^{2} \rightarrow \mathbb{T}^{2}$ such that any liftings $a_{1 *}, a_{2 *}$ of the $a_{1}$, $a_{2}$ to the covering torus satisfy $a_{1 *}{ }^{\circ} a_{2 *}=1$.

ii. If card $\left(a_{1} \cap a_{2}\right)>a_{1} \circ a_{2}$ then for any covering map $\mathbb{T}^{2} \rightarrow \mathbb{T}^{2}$ some liftings $a_{1 *}$, $a_{2 *}$ satisfy card $\left(a_{1 *} \cap a_{2 *}\right)>a_{1 *} \circ a_{2 *}$.

The proof is given in the appendix. Using this lemma we may assume without loss of generality that $a_{1}{ }^{\circ} a_{2}=1$. Then it follows from Lemma 4.5 and 4.6 that the numbers $l^{+}\left(C_{1, \mu}, C_{2}\right)$ are equal to 0 or 1 for each $\mu \in\left[\mu_{-}, \mu_{+}\right] \cap \mathbb{Z}$. On the other 
hand one can easily check that

$$
l^{+}\left(C_{1, \mu}, C_{2}\right)=\sum_{\mu \leqq i \leqq \mu_{+}}(-1)^{i} d_{i}
$$

for each $\mu \in\left[\mu_{+}, \mu_{-}\right] \cap \mathbb{Z}$. We know that $d_{\mu_{+}}>0$ and hence from the first equation where $\mu=\mu_{+}$we obtain $d_{\mu_{+}}=1$.

Lemma 4.9. Let $D=\cup A_{i}, i=0, \ldots, d$, where $A_{i}$ are indexed in accordance with the orientation on the curve $a_{1}, A_{d}=A_{0}$. Then $\left|\mu\left(A_{i+1}\right)-\mu\left(A_{i}\right)\right| \leqq 1$.

The proof is given in the appendix. From this lemma and (4.8) where $l^{+}\left(C_{1, \mu}, C_{2}\right)$ equals 0 or 1 we have that $d_{\mu}=1$ for each $\mu \in\left[\mu_{-}, \mu_{+}\right] \cap \mathbb{Z}$. But this implies that the function $\mu\left(A_{i}\right)$ is strictly monotone. Hence $d=1$. This completes the proof of the theorem.

Proof of Theorem 4.1. If $L_{\omega} \neq G_{\omega}$ then it follows from Theorems 2.4 and 3.6 that the sets $L, G_{\omega}, \sigma(L), \sigma\left(G_{\omega}\right)$ are disjoint. Also there exists a pair of points $O \in G_{\omega}$, $P \in L_{\omega}$ such that some liftings to the covering plane $\mathbb{R}^{2}$ of the geodesics $\theta\left(h_{t} O\right)$ and $\theta\left(h_{t} P\right)$ cross infinitely often. Let $\varepsilon>0$ be sufficiently small to have that the $\varepsilon$-neighbourhoods $U_{\varepsilon}(L), U_{\varepsilon}\left(G_{\omega}\right), U_{\varepsilon}(\sigma(L)), U_{\varepsilon}\left(\sigma\left(G_{\omega}\right)\right)$ are disjoint and $U_{\varepsilon}(L)$ is homeomorphic to $L \times(-\varepsilon, \varepsilon)$. Since $O \in G_{\omega}, P \in L_{\omega}$ then there exists sequences $t_{k} \rightarrow+\infty, s_{j} \rightarrow+\infty$ such that $h_{t_{k}} P \in U_{\varepsilon}(P), h_{s_{j}} O \in U_{\varepsilon}(O)$. Let us consider two smooth closed curves $z_{1}, z_{2}$ in $M$ consisting of the trajectory segments $h_{t} P, t \in\left[0, t_{k}\right], h_{s} O$, $s \in\left[0, s_{j}\right]$, and small arcs joining the ends of these segments in $U_{\varepsilon}(P)$ and $U_{\varepsilon}(O)$ respectively. In addition, we may assume without loss of generality that $z_{1}, z_{2}$ have no self-intersections and the tangent vector to $z_{1}, z_{2}$ at each point is close enough to the hamiltonian vector at the point. Then $\theta\left(z_{1}\right), \theta\left(z_{2}\right)$ are smooth closed curves on $\mathbb{T}^{2}$ such that $\operatorname{leg}\left(\theta\left(z_{1}\right)\right) \subset U_{\varepsilon}(L), \operatorname{leg}\left(\theta\left(z_{2}\right)\right) \subset U_{\varepsilon}\left(G_{\omega}\right)$. Hence it is easily seen that all liftings of the curves $z_{1}, z_{2}, \sigma\left(z_{1}\right), \sigma\left(z_{2}\right)$ to the covering space $N$ are smooth non-intersecting closed curves and any two of them are unlinked. In addition we may assume that $\theta\left(z_{1}\right)$ and $\theta\left(z_{2}\right)$ are transversal and have only transversal self-intersections. It follows from Theorem 4.1 of [B-P2] that $\theta\left(z_{1}\right)$ and $\theta\left(z_{2}\right)$ have no contractible loops on $\mathbb{T}^{2}$, and hence using Proposition 5.2 of [B-P2] we may assume that $\theta\left(z_{1}\right)$ and $\theta\left(z_{2}\right)$ are smooth simple closed curves on $\mathbb{T}^{2}$. On the other hand, it follows from Theorem 3.6 that some liftings of $\theta\left(z_{1}\right)$ and $\theta\left(z_{2}\right)$ to the covering plane $\mathbb{R}^{2}$ cross more than once and hence card $\left(\theta\left(z_{1}\right) \cap \theta\left(z_{2}\right)\right)>$ $\left|\theta\left(z_{1}\right) \circ \theta\left(z_{2}\right)\right|$. But this is a contradiction with the result of Theorem 4.4.

\section{Proof of the Main Theorem}

It follows from Theorem 4.1 that each recurrent trajectory on $L$ projects to an $A$-geodesic on $\mathbb{T}^{2}$. Let us show now that each trajectory on $L$ projects to an $A$-geodesic. Let $P \in \gamma-\gamma_{\omega}$, and $h_{t} P \cap \gamma=\cup P_{n}, n \in \mathbb{Z}$ (see Theorem 2.4). There exists $Q \in \gamma_{\omega}$ such that $P_{n}$ and $Q_{n}$ are asymptotic when $n \rightarrow \pm \infty$. Let $p_{n}=\theta\left(P_{n}\right), q_{n}=\theta\left(Q_{n}\right)$. Let $F\left(p_{-n}, p_{n}\right)$ be the $F$-length of the geodesic $\theta\left(h_{t} P\right)$ between the points $p_{-n}, p_{n}$, the number $F\left(q_{-n}, q_{n}\right)$ is defined analogously. Let us denote by $f\left(p_{-n}, p_{n}\right)$ the minimum $F$-length of all segments connecting $p_{-n}, p_{n}$ homotopic to the segment 
of $\theta\left(h_{t} P\right)$ between these points, we define $f\left(q_{-n}, q_{n}\right)$ analogously. It follows easily from the definition of the metric $F$ that

$$
2 F\left(q_{-n}, q_{n}\right)=\int_{Q-n Q_{n}} \omega^{1}, \quad 2 F\left(p_{-n}, p_{n}\right)=\int_{P-n} \omega_{n} \omega^{1},
$$

where $Q_{-n} Q_{n}$ and $P_{-n} P_{n}$ are trajectory segments of $h_{t} Q, h_{t} P$ between the points $Q_{-n}, Q_{n}$ and $P_{-n}, P_{n}$ respectively. Also $F\left(q_{-n}, q_{n}\right)=f\left(q_{-n}, q_{n}\right)$ since $\theta\left(h_{t} Q\right)$ is an $A$-geodesic. If $\theta\left(h_{t} P\right)$ is not an $A$-geodesic, then for some constant $f>0$ for all $n>n_{0}$

$$
F\left(p_{-n}, p_{n}\right)>f\left(p_{-n}, p_{n}\right)+f \text {. }
$$

For a small $\varepsilon>0$ let us choose $n>n_{0}$ such that the segment $\delta_{n}$ of $\gamma$ between the points $P_{n}, Q_{n}$ lies in an $\varepsilon$-ball $U_{n}$, also the segment $\delta_{-n}$ of $\gamma$ between the points $P_{-n}$, $Q_{-n}$ lies in an $\varepsilon$-ball $U_{-n}$. Let $\delta_{-n *}$ be a smooth simple arc in $U_{-n} \cap S$ with the ends $P_{-n}, Q_{-n}$ approximating $\delta_{-n}(S$ is defined in Sect. 2). It follows from Theorem 2.4 and the theorem of continuous dependence on initial data that there exists a smooth function $\tau: \delta_{-n *} \rightarrow \mathbb{R}$, such that

$$
h_{\tau(Q-n)} Q_{-n}=Q_{n}, \quad h_{\tau(P-n)} P_{-n}=P_{n} \quad \text { and } \quad h_{\tau(x)} x \in S \cap U_{n} \quad \text { for all } x \in \delta_{-n *} .
$$

In addition, the arc

$$
\delta_{n *}=\left\{y: y=h_{\tau(x)} x \text { for some } x \in \delta_{-n *}\right\}
$$

is well defined and without self-intersections. It follows from Stokes' theorem that

$$
2 F\left(p_{-n}, p_{n}\right)-2 F\left(q_{-n}, q_{n}\right)=\int_{\delta-n *} \omega^{1}-\int_{\delta_{n *}} \omega^{1} .
$$

Both of the integrals on the right-hand sides are less than const $\cdot \varepsilon$, since $\delta_{-n *}$, $\delta_{n *} \subset S$ are simple arcs lying in the $\varepsilon$-balls $U_{-n}, U_{n}$. This implies that

$$
2 f\left(q_{-n}, q_{n}\right)=2 F\left(p_{-n}, p_{n}\right)-\text { const } \cdot \varepsilon>2 f\left(p_{-n}, p_{n}\right)+f-\text { const } \cdot \varepsilon .
$$

But the last inequality means that the geodesic $\theta\left(h_{t} Q\right)$ is not minimal between the points $q_{-n}, q_{n}$. This contradiction yields the proof of the fact that the projections on $\pi^{2}$ of the trajectories of the flow $h_{t}$ on $L$ are $A$-geodesics. Now it follows from Theorem 3.5(i) that none of these $A$-geodesics intersect each other. This implies that $L$ is a cross-section of the cotangent bundle $T^{*} \pi^{2}$. This completes the proof of the main theorem.

Remark 5.1. Using the same method one can prove that in the case when $L$ contains closed orbits of the flow, projections of these orbits on $\mathbb{T}^{2}$ are $A$-geodesics. For this one needs the classification theorem of $K_{s}, \Gamma_{s}$ for rational $s$ (see [B-P1]).

\section{Appendix. Proof of Lemmas 4.7, 4.9.}

\section{Proof of Lemma 4.7.}

i. Let $\left(m_{1}, n_{1}\right),\left(m_{2}, n_{2}\right)$ from $\mathbb{Z}^{2}$ represent homotopy classes of $a_{1}, a_{2}$ on $\mathbb{T}^{2}=\mathbb{R}^{2} / \mathbb{Z}^{2}$. Let $G \subset \mathbb{Z}^{2}$ be the subgroup generated by these elements. Then the natural covering map $\mathbb{R}^{2} / G \rightarrow \mathbb{R}^{2} / \mathbb{Z}^{2}$ is the desired covering map. 
ii. We have assumed that $\operatorname{card}\left(a_{1} \cap a_{2}\right)>a_{1} \circ a_{2}>0$. This implies that there is a point $A \in a_{1} \cap a_{2}$ such that the frame $\left(\dot{a}_{1}, \dot{a}_{2}\right)$ is negative at $A$. Let $A_{*}$ be some lifting of $A$ to the covering torus, $a_{1 *}, a_{2 *}$ the liftings of $a_{1}, a_{2}$ passing through $A_{*}$. Then the frame $\left(\dot{a}_{1 *}, \dot{a}_{2 *}\right)$ is negative at $A_{*}$, and hence card $\left(a_{1 *} \cap a_{2 *}\right)>a_{1 *} a_{2 *}$. This completes the proof.

Proof of Lemma 4.9. Let $A_{i}, A_{i+1}$ from $a_{1} \cap a_{2}$ be such that $A_{i+1}$ is the next point after $A_{i}$ on $a_{1}$ with respect to the orientation on $a_{1}$. Let $A_{i *}$ be some lifting of $A_{i}$ to the covering plane, $a_{1 *}, a_{2 *}$ the liftings of $a_{1}, a_{2}$ passing through $A_{i *}$. Let $A_{i+1 *}$ be the lifting of $A_{i+1}$ belonging to $a_{1 *}$. We have that $a_{2 *}$ is a simple periodic curve homeomorphic to $\mathbb{R}$, also different liftings of $a_{2}$ do not intersect. We may assume without loss of generality that $T_{2}\left(a_{2 *}\right)=a_{2 *}\left(T_{1}, T_{2}\right.$ are the unit shofts of $\mathbb{R}^{2}$ along the axes $q_{1}, q_{2}$ respectively). It follows from the definition of $A_{i+1}$ that the open segment of $a_{1 *}$ between the points $A_{i *}, A_{i+1 *}$ has no intersections with $\left(T_{1}\right)^{n}\left(a_{2 *}\right)$ for each $n \in \mathbb{Z}$. Let us consider two cases:

1. $A_{i+1 *} \in a_{2 *}$ and 2. $A_{i+1 *} \notin a_{2 *}$.

1. In this case the frame $\left(\dot{a}_{1 *}, \dot{a}_{2 *}\right)$ has different signs at the points $A_{i *}, A_{i+1 *}$. Let us assume for example that this sign at $A_{i *}$ equals -1 (the opposite case is analogous). It follows from the definition of the functions $\varphi_{1}, \varphi_{2}$ that

$$
\varphi_{1}\left(A_{i+1}\right)-\varphi_{1}\left(A_{i}\right)=\delta \varphi_{1}, \quad \varphi_{2}\left(A_{i}\right)-\varphi_{2}\left(A_{i+1}\right)=\delta \varphi_{2},
$$

where $\delta \varphi_{1}$ is the rotation of the tangent vector to $a_{1 *}$ from $A_{i *}$ to $A_{i+1 *}, \delta \varphi_{2}$ is the rotation of the tangent vector to $a_{2 *}$ from $A_{i+1 *}$ to $A_{i *}$. Also the oriented angle from $\dot{a}_{1 *}$ to $\dot{a}_{2 *}$ equals $\pi+v\left(A_{i}\right)$ at $A_{i *}$ and $v\left(A_{i+1}\right)$ at $A_{i+1 *}$. It follows from [St, p. 261] that

and hence

$$
\delta \varphi_{1}+\delta \varphi_{2}=2 \pi-v\left(A_{i+1}\right)-\left(\pi-v\left(A_{i}\right)\right)
$$

and hence

$$
\mu\left(A_{i}\right) \pi+v\left(A_{i}\right)-\mu\left(A_{i+1}\right) \pi-v\left(A_{i+1}\right)=\pi-v\left(A_{i+1}\right)+v\left(A_{i}\right)
$$

$$
\mu\left(A_{i}\right)-\mu\left(A_{i+1}\right)=1 .
$$

2. In this case analogous arguments show that $\mu\left(A_{i+1}\right)-\mu\left(A_{i}\right)=0$. This completes the proof of the lemma.

Acknowledgements. I am grateful to Professor Y. Yomdin for his interest in this work, and to R. MacKay for reading the manuscript and for useful discussion.

\section{References}

[Ar] Arnold, V. I.: Mathematical methods of classical mechanics. Berlin, Heidelberg, New York: Springer c 1978

[A] Aubry, S., Le Daeron, P. Y.: The discrete Frencel-Kontorova model and its extension. Physica 8D, 381-422 (1983)

[Ba] Bangert, V.: Mather sets for twist maps and geodesics on tori. Dynamics reported 1 (1987)

[Be] Beck, A.: continuous flows in the plane. Berlin, Heidelberg, New York: Springer 1974

[B] Bialy, M.: On the number of caustics for invariant tori of hamiltonian systems with two degrees of freedom. To appear in Ergodic Theorem Dyna. Syst. 
[B-P1] Bialy, M. L., Polterovich, L. V.: Geodesic flows on the two-dimensional torus and phase transitions "commensurability-noncommensurability". Funct. Anal. Appl. 20, 260-266 (1986)

[B-P2] Bialy, M. L., Polterovich, L. V.: Lagrangian singularities of invariant tori of hamiltonian systems with two degrees of freedom. To appear in Inv. Math. 1989

[C-L] Coddington, E., Levinson, N.: Theory of ordinary differential equation. New York: McGraw-Hill 1955

[E] Edwards, C. H.: Concentric solid tori in the 3-sphere. Trans. Am. Math. Soc. 102, 1-17 (1962)

[Hed] Hedlund, G.: Geodesics on a two-dimensional Riemannian manifold with periodic coefficients. Ann. Math. Ser. 2, 33, 719-739 (1932)

[H1] Herman, M.: Sur les courbes invariantes par les diffeomorphisms de l'anneau. Asterisque 103-104 (1983)

[H2] Herman, M.: Existence et non existence de tores invariants par des diffeomorphismes symplectiques. Preprint (1988)

[M] Mather, J.: A criterion for the non-existence of invariant circles. Publ. Math. I.H.E.S. 63, 153-204 (1986)

[Mu Munkres, J.: Obstructions to the smoothing of piecewise-differentiable homeomorphisms. Ann. Math. Ser. 2, 72, 521-554 (1960)

[Ru] Rushing, B.: Topological Embeddings. New York, London: Academic Press 1973

[St] Sternberg, Sh.: Lectures on differential geometry. New Jersey: Prentice-Hall 1964

Communicated by J. N. Mather

Received November 1, 1988; in revised form May 10, 1989 